\title{
Estudio Computarizado de Morbilidad y Mortalidad en Recién Nacidos
}

\author{
Dra. Elizabeth Weldt S. 1: Dr. Enrique Hering A. ${ }^{1}$
}

\author{
Computerized Study of Morbi-Mortality in a Special \\ Care Baby Unit
}

\begin{abstract}
A computerized study of morbility and mortality in a Special Carc Baby Unit was done along a period of five months, from January to May 1982. Among 4644 newborns, 743 (15.9\%) were admited to the Unit because of low birth weight (less than $2.000 \mathrm{~g}$.) or other type of illness. The study showed that $24,5 \%$ of the netvborn had a birth weight less than $2.500 \mathrm{~g}$. and that the gestational age was less than 37 weeks in $27,6 \%$ The most frecuent diseases were jaundice $(86,4 \%)$, infections $(16,7 \%)$ and respiratory distress sindrome $(9,3 \%)$. The mortality rate was $9,4 \%$ and the lethality $5,9 \%$ The most afected group was that with birth weight less than $1.500 \mathrm{~g}$. Death tates were $88 \%$ for newborns of $1.000 \mathrm{~g}$. or less and $20 \%$ in those weighing from 1.001 to $1.500 \mathrm{~g}$. at birth. This study allows us to know the main pathological problems in this group of patients and by means of it, to find adecuatc policies to face them.
\end{abstract}

La dificultad para obtener una información actualizada de la morbimortalidad neonatal en una Maternidad con gran volumen asistencial (12.000 partos al año), y con una cifra aproximada de 2.500 hospitalizaciones neonatales, nos ha motivado a obtenerla a partir de un estudio computarizado de los datos clínicos de los recién nacidos (R.N.) de la Unidad de Neonatología de la Maternidad del Salvador. Conocer las afecciones más frecuentes de los RN en nuestra Unidad, y las principales causas de muerte, resulta de gran importancia descle el punto de vista estadístico y administrativo, pues ayuda a adecuar recursos, modificar conductas y evaluar la nueva dotación de equipos para la atención perinatal.

\section{MATERIAL, Y METODO}

Entre Enero y Mayo de 1982, nacieron en la Maternidad del Salvador 4.644 nirros, de los cuales 743 fueron ingresados a la Unidad de Neonatología (U.N.) por tener un peso inferior a $2.000 \mathrm{~g}$., presentar algún tipo de patología o ambas razones.

Se confeccionó una ficha que registra la identificación del R.N., sus antecedentes familiares, del embarazo y parto, diagnósticos, tratamientos, condiciones de alta, días de estada y alimentación recibida.

La ficha se procesó en un computador IBM 370 de la Facultad de Ciencias Físicas y Matemáticas de la Universidad de Chile, empleando un lenguaje diseñado para apoyar la realización de análisis estadísticos (SPSS).
Los pacientes del estudio fueron agrupados según Peso de Nacimiento (PN), Edad Gestacional (EG) y Crecimiento Intrauterino. De acuerdo con la edad gestacional, se dividió el grupo estudiado en niños đe pretérmino, término y post término. Según el Crecimiento Intrauterino se les calificó como pequeño, adecuado y grande para la edad gestacional. Para la clasificación de Crecimiento Intrauterino se utilizaron las curvas de Lubchenco.

La morbilidad observada se clasificó en Ictericia, Infecciones, Sindrome de Dificultad Respiratoria (S.D.R.), Trastornos Hematológicos, Malformaciones Congénitas, Trastornos Metabólicos, Traumatismos del Parto, Alteraciones del Sistema Nervioso Central, Infección Ovular, Apneas, Infección Connatal y Problemas Mater. nos.

Las Ictericias se dividieron en Incompatibilidad de grupo clásico $(\mathrm{AB} / \mathrm{O})$, Incompatibilidad Rh a Hiperbilirrubinemias Idiopáticas, que corresponden a aquellos casos sin Incompatibilidad sanguínea.

Las Infecciones se clasificaron en Menores (onfalitis, conjuntivitis, piodermitis, rinitis), Ma. yores (septicemias, bronconeumonias, meningitis, osteortritis) y Diarreas.

En Sindrome de Dificultad Respiratoria se incluyeron el Síndrome de Dificultad Respiratoria Idiopática (SDRI), la Taquipnea Transitotia (TT), el Sindrome de Broncoaspiración, la Bronconeumonia Connatal, el Neumatórax Espontáneo y la A telectasia Pulmonar.

En los trastornos Hematológicos se consignaron aquellos casos de Anemia con valores $\mathbf{H b}$, que requirieron de transfusión, Policitemia cuando el hematocrito fue mayor de $65 \%$ y Sindrome Hemorrágico. 
Las Malformaciones congénitas se clasificaron por sistemas.

En trastornos metabólicos se consideraron las Hipoglicenias, Hipocalcemias y Acidosis Metabólica tardía del prematuro.

Los traumatismos del parto, compreden las Fracturas de Clavícula, Cráneo, Húmero y Fémur, Cefalohematoma, Hematoma Subaponeurótico y Equimosis múltiples.

Entre las alteraciones del S.N.C. se incluyeron los trastornos de la función Neurológica con especificación etiológica cuando ello fue posible a través de Ultrasonografía y/o Autopsia.

\section{RESULTADOS}

En la Tabla 1, se muestra la distribución de 743 Recién nacidos Hospitalizados, según peso de nacimiento. $17(2,3 \%)$ pesaron menos de $1.000 \mathrm{~g}$. El total de Recién Nacidos con peso comprendido entre 1.001 y $2.500 \mathrm{~g}$, fue 165 $(22,2 \%)$ y sobre $2.500 \mathrm{~g}$., hubo $561(75,5 \%)$.

Tabla 1.

Distribución de 743 Reclén Nacidas Hospitalizados según peso de Nacimiento

\begin{tabular}{crr} 
& \multicolumn{1}{c}{$\mathrm{N}^{\circ}$} & $\%$ \\
$-1.000 \mathrm{~g}$. & 17 & 2,3 \\
$1.001-1.500 \mathrm{~g}$. & 25 & 3.3 \\
$1.501-2.000 \mathrm{~g}$. & 60 & 8,1 \\
$2.001-2.500 \mathrm{~g}$. & 80 & 10,8 \\
$2.501-3.000 \mathrm{~g}$. & 157 & 21,2 \\
$3.001-3.500 \mathrm{~g}-$ & 218 & 29,3 \\
$3.501-4.000 \mathrm{~g}$. & 147 & 19.8 \\
$+4.000 \mathrm{~g}$. & 39 & 5,2 \\
\hline Total & 743 & 100,0
\end{tabular}

La Edad Gestacional (EG) fue inferior a 37 semanas en $27,5 \%$ de los RN, en $59,4 \%$ fluctuó entre 38 y 42 semanas $y$ sólo tuvimos 1 niño con EG mayor que 42 semanas. En 96 ingresos $(12,9 \%)$ del grupo estudiado, la EG no se registró por no coincidir la fecha de última regla (FUR) con la evaluación pediátrica.

Los RN pretérmino fueron 204, de ellos $87,2 \%$ fueron AEG, 11,2\% PEG y 1,4\% GEG. Los RN de término fueron 442 con $89 \%$ AEG, $2 \%$ PEG y $8 \%$ GEG (Figura 1). Se excluyó de la Figura 1 un RN post término y los $96 \mathrm{RN}$ cuya EG fue incierta.

En la Tabla 2, se muestra en orden de frecuencia la morbilidad encontrada en estos RN:

El problema más frecuente fue la ictericia que ocurrió en 642 casos: En $77,29 \%$ no habia incompatibilidad sanguínea, en $21 \%$ existía incompatibilidad de grupo clásico y en $1,62 \%$ se trataba de enfermedad hemolítica por incompatibilidad Rh.

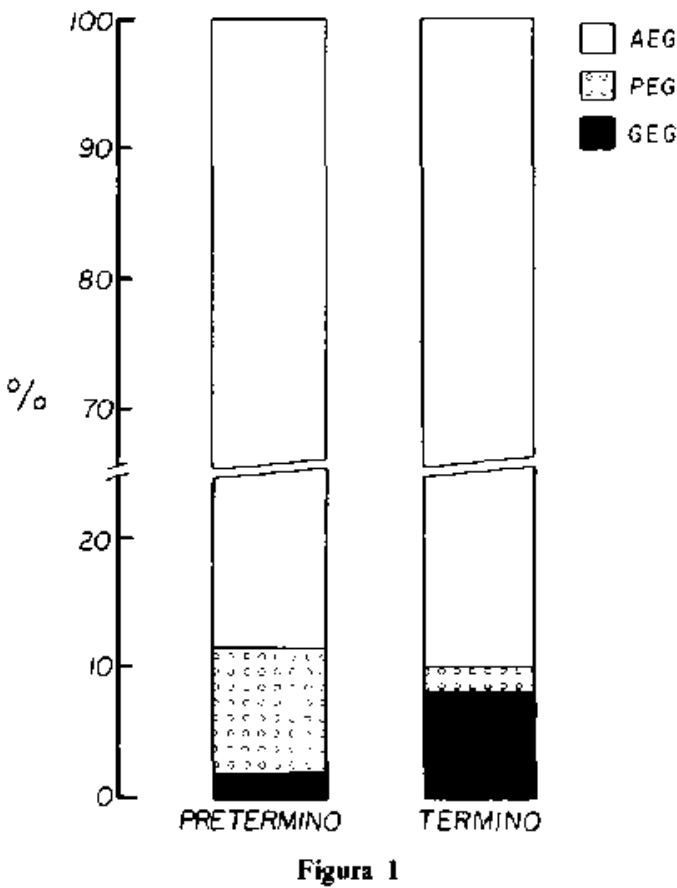

Distribución đe los recién nacidos según tamaño para la Edad Gestacional (EG): Adecuado (A); Pequeño (P) y Grande (G)

Tabla 2.

Morbilidad en 743 RN Hospitalizados

$\begin{array}{lrr}\text { Diagnóstico } & \text { No } & \% \\ \text { Ictericia } & 642 & \mathbf{8 6 , 4} \\ \text { Infecciones } & 124 & \mathbf{1 6 , 7} \\ \text { S.D.R. } & 69 & \mathbf{9 , 3} \\ \text { Problemas hematológicos } & 69 & 9,3 \\ \text { Malformaciones congénitas } & 55 & \mathbf{7 , 4} \\ \text { Problemas metabólicos } & 41 & 5,5 \\ \text { Traumatismo del parto } & 41 & 5,5 \\ \text { Alteraciones del S.N.C. } & 29 & 3,9 \\ \text { Infección ovular } & 25 & 3,4 \\ \text { Apneas } & \mathbf{1 8} & 2,4 \\ \text { Problemas maternos } & 14 & 1,9 \\ \text { Infección connatal } & 4 & 0,5\end{array}$

La segunda frecuencia corresponde a las infecciones, que ocurrieron en 124 casos $(16,7 \%)$. 54 RN tenían infecciones menores, predominando onfalitis y conjuntivitis, cuya causa más frecuente fue Staphylococcus Aureus; En 34 RN se diagnosticó algún tipo de infección mayor (19 septicemias, 11 bronconeumonias, 3 meningitis y 1 infección urinaria). En el $58 \%$ de los cultivos positivos entre estos pacientes, el germen aislado 
fue una Klebsiella. 36 RN sufrieron Diarrea Aguda causada por E. coli enteropatógeno cepa 0143.

El Sindrome de Dificultad Respiratoria (SDR) ocupa el tercer lugar con 69 casos de los cuales $46,4 \%$ fueron catalogados como S.D.R. Idiopático, $29 \%$ como Taquipnea transitoria y $14,5 \%$ como Sindrome de aspiración, además habían 4 casos de bronconeumonias connatales, 2 con Neumotórax Espontáneos y 1 de Atelectasia del Lóbulo Medio Derecho.

Los problemas hematológicos, se presentaron en 69 recién nacidos de los cuales 44 correspondieron a anemia y 29 a policitemia de acuerdo a la defirición previamente establecida.

La mayor incidencia de malformaciones congénitas correspondió a las cardiopatías congénitas con 21 casos y las anomalías cromosómicas en $10 \mathrm{RN}$. Los problemas metabólicos principales fueron: Hipoglicemia $(n=26)$, Acidosis metabólicas tardías del prematuro $(n=13)$ e Hipocalcemia $(n=2)$.

En los traumatismos del parto predominaron las cefalohematomas con 20 casos y 11 fracturas de claviculas.

En 29 recién nacidos, se diagnosticó un Sindrome de Disfunción Neurológica, en 17 de ellos se demostró que había una Hemorragia intracraneana.

La causa de ingreso fue Apnea en 18 pacientes, 13 fueron calificadas como secundarias y 5 primarias.

Catorce recién nacidos fueron ingresados por enfermedad de la madre.

En el grupo de peso inferior a $1.000 \mathrm{~g}$. la sobrevida fue del $12,6 \%$ mejorando considerablemente a mayor peso, para tener una sobrevida alrededor del $90 \%$ sobre 1.500 g. (Figura 2).

En la Tabla 3 se muestran las causas de muerte de los $44 \mathrm{RN}$ fallecidos. La prematurez, el Sindrome de difjcultad respiratoria idiopático, las cardiopatias congénitas, la hemorragia intracraneana y la septicemia fueron las causas más frecuentes en esta serie.

\section{DISCUSION}

La información obtenida por computadoras de la morbimortalidad neonatal, en el nivel local permite tener una visión global de los principales problemas que afectan a este grupo etáreo y evaluar en forma dinámica el rendimiento de los Programas Perinatales 1.

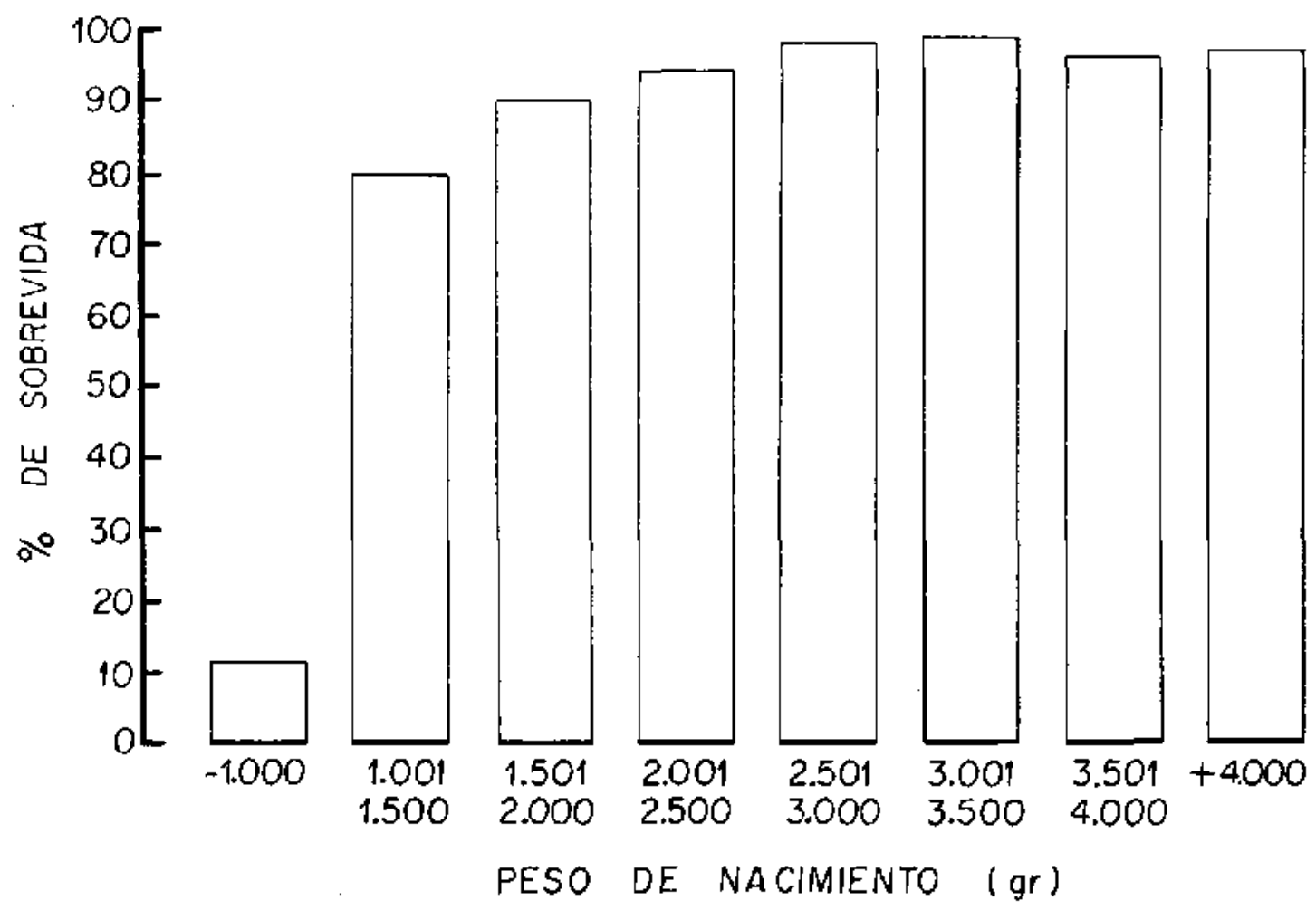

Figura 2

Sobrevida de los recién nacidos según peso al nacer. 
Tabla 3.

Distribución de 44 RN fallecidos de acuerdo a causa de mucrte

Causa de Muerte

No

Prematuridad extrema:

Malformaciones cogénitas:

-- Cardiopatías congénitas

- Malformaciones múltiples

- Genopatias

- Malfomaciones S.N.C.

- Hernia diafragmática

Sindrome de dificultad respiratoria:

- S.D.R.I.

- Aspiración meconial

- Aspiración de leche

Hemotragia in tracraneana

Septicemia

Hidrops por

incompatibilidad $\mathrm{RH}$

Toxoplasmosis congénita:

Total

44

Las cifras obtenidas en este estudio nos mues tran que nuestro principal esfuerzo debe dirigirse a la atención del RN de bajo peso, que requiere de observación y cuidados especiales, pues en él se concentran mayores morbilidad y mortali. $\operatorname{dad}^{2-3-4}$.

El mejor estudio de la Unidad Feto Placentaria, con parámetros bioquímicos de líquido anuniótico y ecografías explican la baja incidencia de desnutrición intrauterina y de RN post término.

Llamó la atención la alta incidencia de ictericia que constituyó el problema más frecuente. La mayoría de los casos correspondieron a hi. perbilirrubinemia idiopática, lo que nos obligará a hacer estudios posteriores para tratar de pesquisar los factores etiológicos y explicar su frecuencia.

Las infecciones ocuparon el segundo lugar con una frecuencia alta, sin embargo debemos aclarar que durante el período de estudio se produjo un brote epidémico de diarrea por E. coli $0143^{5}$. que explicaría en parte la mayor incidencia además de otros factores como mejor sobrevida de $\mathrm{RN}$ de muy bajo peso, aumento de la instru. mentación, técnicas de manejo más agresivas (como ventilación mecánica) y limitación de los recursos disponibles, todos los que contribuyen a aumentar el riesgo de infección intrahospitalaria ${ }^{6-7}$

Es importante también destacar el hallazgo de Klebsiella como agente causal en un número importante de casos con infecciones. Este germen tha mostrado, en el nivel local, resistencia a los antibióticos de uso habitual (gentamicina), obligando a introducir nuevos antibióticos, reforzando la necesidad de una constante vigilancia epidemiológica en cada Unidad, para identificar la flora predominante y conocer su sensibili$\operatorname{dad}^{8-9}$.

Los trastomos respiratorios tuvieron una incidencia global del 9,3\%. El S.D.R.I. ocurrió en 32 casos, to que representa una incidencia real, en los RN de pre-término del grupo estudiado de $16 \%$. Esto es de extraordinaria importancia, pues la proporción eta en años anteriores de $25 \%$ a $30 \%$. Es muy posible que la disminución del número de casos refleje mejor control y manejo del embarazo de alto riesgo, vigilancia adecuada de la madurez pulmonar e inducción de ella cuando los factores mórbidos matemos to requieren 10 .

Por otra parte, la existencia de $10 \mathrm{RN}$ con broncoaspiración masiva, que representa un $4,5 \%$ del total de RN con S.D.R. es una cifra alta, susceptible de ser disminuida significativamente mediante la aspiración intraparto rutinaria, en aquellos casos con liquido amniótico con meconio, por lo cual se deberá insistir tanto en el procedimiento mismo, como en la importancia del trabajo coordinado del equipo obstétrico neonatal.

Llanó la atención la incidencia de problemas hematológicos. En 44 casos se diagnosticó anemia que requirió ser corregida con una o más transfusiones. Esto coincidió en general con RN de alto riesgo que por su morbilidad requirieron de numerosas y repetidas muestras sanguíneas para examenes. No se consideraron en el recuento casos de anemia "fisiológica" del prematuro, cuyas cifras de hemoglobina se mantuvieran en cifras aceptables segun las curvas de Oski. En 20 RN se diagnostico Policitemia, coincidiendo en la mayoría de los casos con niños que presentaban una desnutrición intrauterina.

En $7,4 \%$ de los pacientes se encontraron malformaciones congénitas, predominando las alteraciones del Sistema Cardiovascular y Enferme. dades genéticas. Es un hecho conocido que en la medida que se logra disminuir la tasa de infeccio. nes y enfermedades respiratorias, las malformaciones adquieren mayor relevancia. Vale la pena destacar que si bien la incidencia de malformaciones es superior a la comunicada por otros auto. res ${ }^{11}$ ello se debe a que esta muestra es seleccionada pues consideró RN hospitalizados y no el total de niños vivos.

La incidencia de $5,5 \%$ de trastornos metabóli$\cos$ de los que casi $2 / 3$ corresponden a Hipoglicemia, está de acuerdo con las cifras nacionales ${ }^{12}$ para esta afección y se concentró especialmente 
en el grupo de recién nacidos de bajo peso y/o desnutridos intrauterinos.

Los neonatos que presentaron algún tipo de compromjso neurológico en el período de $\mathrm{RN}$ inmediato corresponden a $3,9 \%$ de los hospitalizados. Es importante destacar que en 17 casos $(58,8 \%)$ se logró determinar con precisión la existencia de una hemorragia intracraneana periventricular o peribulbar, mediante ultrasonografía encefálica13.14, método de exploración no invasivo que ha adquirido mayor importancia al demostrarse su utilidad en estos problemas. Esta ténica junto con la tomografía computarizada debería permitir en el futuro, definir con mayor exactitud $y$ en un mayor número de casos, el componente orgánico de los síndromes de disfunción neurológica.

En el grupo estudiado se encontró $2,4 \%$ de episodios de apnea, de los cuales $2 / 3$ fueron catalogados como secundarios a hemorragia intracraneana, trastornos metabólicos o ambos, lo que significa que una investigación dirigida permite un diagnóstico más preçiso, corregir la causa cuando es posible o tener mejores fundamentos para el tratamiento, evitando el manejo empírico, que puede resultar de mayor riesgo, como podría sec el empleo rutinario de xantinas en cualquier tipo de apnea neonatal.

El indice de letalidad global del grupo estudiado fue de $5,9 \%$. Cabe destacar en primer término que ella incluye el recién nacido de menos de $1.000 \mathrm{~g}$, factor evidentemente importante de considerar cuando se quiere medir el impacto que este grupo etáreo tiene en el índice. De $43 \mathrm{RN}$ fallecidos, $40 \%$ correspondió a niños de un peso inferior a $1.000 \mathrm{~g}$.

Las causas de muerte más frecuentes fueron los trastornos respiratorios, las malformaciones congénitas y las hemorragias intracraneanas.

En lo que se refiere al diagnóstico de "prematuridad extrema", que es aceptada intemacionalmente $y$ en la mayoria de los casos corresponde a prematuros de peso inferior a I k., será necesario mejorar, en el futuro, el estudio anatopatológico y tratar de precisar mejor la causa del fallecimiento.

\section{RESUMEN}

Se efectúa un estudio computarizado para conocer la Morbimortalidad en la Unidad de Neonatología de la Matemidad del Hospital Sal. vador. Durante el período en estudio (5 meses) nacen 4.644 niños de los cuales $743(15,9 \%)$ se ingresan a la Unidad de Neonatología por tenet un peso de nacimiento inferior a $2.000 \mathrm{~g}$. o presentar algún cuadro mórbido.

El estudio demostró que $24,5 \%$ de los recién nacidos ingresados a la Unidad pesó menos de $2.500 \mathrm{~g}$. y que la edad gestacional fue inferior a 37 semanas en $27,5 \%$.

Las morbilidades más frecuentes fueron: Ictericia $(86,4 \%)$, Infecciones $(16,7 \%)$ y sindrome de Dificultad Respiratoria $(9,3 \%)$.

La tasa de mortalidad fue $9,4 \%$ o y la letalidad 5,9\%, el grupo más afectado fue aquel con peso de nacimiento inferior a $1.500 \mathrm{~g}$., con $88 \%$ para el menor de $1.000 \mathrm{~g}$ y $20 \%$ entre $1.001 \mathrm{y}$ $1.500 \mathrm{~g}$.

La evaluación computacional de la morbimortalidad neonatal resulta de extraordinario interés tanto por la posibilidad de conocer rápidamente los problemas prioritarios y por ende adecuar los recursos para su manejo, como por la alternativa de combinar distintos parámetros que nos permite conocer morbimortalidad por causa específica, peso al nacer y desarrollo intrauterino que es muy difícil definir cuando se trabaja con un promedio de 12.000 partos anuales.

\section{AGRADECIMIENTOS}

Este proyecto fue posible de realizar gracias a la valiosa y desinteresada cooperación del Sr. Juan Alvarez R., Profesor de Computación de la Facultad de Ciencias Físicas y Matemáticas de la Universidad de Chile y de la Srta. Evelyn Sandoval P., alumna de Ingeriería de Programación e Informática.

\section{REFERENCIAS}

1 Janik, D., Wyman, M., Computarized newborn intensive care data recording and research III. A practical microcomputer system J. Pediat. 97: 497. 1980.

2 Beveriy L, Kaops Atel: Neonatal Mortality risk in relation to birth weight and gestational age (Up date), J. Pediat. 101: 969, 1982.

3 Valdés-Dopena M.A., Arey J.B.: The causes of neonatal mortality: an analysis of 501 autopsies on newborn infants. J. Pediat. 77: 366, 1970.

4 Usher. $R$.: Clinical implications of Perinatal Mortality Stadistics. Clin. Obst. Gyn, 14: 885, 1971.

5 Hering E., Morombio E., Prado, V.: Análisis de un brote de diarrea por $E$. coli 0142 en recién nacidos. Rey. Clíl. Pediat. 55: 175, 1984.

${ }^{6}$ Marks M. Weldch, D.: Diagnosis of bacterial infections of the newborn infant. Clinics in Perinatology i (3): 537, 1981 .

7 Devies P.: Bacterial infections in the fetus and newborn. Arch. Dis. Child. 46: 1, 1981.

Berkovic P., Geldres V., Beca J.P., Howard J., Román $C_{1}$, Septicemia por Klebsiella enterobacter en recién nacidos. Rev. Chil. Pediat. 50: 37, 1979.

${ }^{9}$ Herskovic P., Toso A., Beca, J.P., Donoso, E. Etiologia de las infecciones bacterianas neonatales. Rev. Chil. Pediat. 50: 42, 1979.

10 Tisné, L. y col.: Extensión del programa de atención integral de la mujer en el área Oriente de Santiago y descenso de la morbimortalidad materna y peri. natal. (1959-1975). 
11 Avery, G.: Neomatology, pathophisiology and manegement of the newborn, Second Edition, J.B. Lippincott Co. Philadelphia, 1982.

12 Beca. J.P., Romen, C.: Hipoglicemia neonatal. Pediatría (Stgo.) 19:212, 1976.

13 Norambuena N., Klein F., Rosselot S., Vaccaro H.,. Diagnóstico de la hemorragia intracraneana mediante ecografía encefálica en recién nacidos de bajo peso (menos $1.500 \mathrm{~g}$ ). Correlación anátomo patoló gica. $14^{\circ}$ Congreso Chileno de Pediatría, Puerto Montt, 1982.

14 Klein, F., Narambuena, N., Rosselot, S., Vaccaro, H., Códiz, H., Martínez, E., Gona, J.: Exploración ecográfica del encéfalo de recién nacidos de término y pre-término. Parámetros de normalidad. $14^{\circ}$ Congreso Chileno de Pediatría. Puerto Montt, 1982. 